\title{
IMMERSED $n$-MANIFOLDS IN $\mathbf{R}^{2 n}$ AND THE DOUBLE POINTS OF THEIR GENERIC PROJECTIONS INTO $\mathbf{R}^{2 n-1}$
}

\author{
OSAMU SAEKI AND KAZUHIRO SAKUMA
}

\begin{abstract}
We give two congruence formulas concerning the number of nontrivial double point circles and arcs of a smooth map with generic singularities - the Whitney umbrellas - of an $n$-manifold into $\mathbf{R}^{2 n-1}$, which generalize the formulas by Szücs for an immersion with normal crossings. Then they are applied to give a new geometric proof of the congruence formula due to Mahowald and Lannes concerning the normal Euler number of an immersed $n$-manifold in $\mathbf{R}^{2 n}$. We also study generic projections of an embedded $n$ manifold in $\mathbf{R}^{2 n}$ into $\mathbf{R}^{2 n-1}$ and prove an elimination theorem of Whitney umbrella points of opposite signs, which is a direct generalization of a recent result of Carter and Saito concerning embedded surfaces in $\mathbf{R}^{4}$. The problem of lifting a map into $\mathbf{R}^{2 n-1}$ to an embedding into $\mathbf{R}^{2 n}$ is also studied.
\end{abstract}

\section{INTRODUCTION}

Let $g: M^{n} \rightarrow \mathbf{R}^{2 n-1}(n \geq 2)$ be an immersion with normal crossings of a closed $n$-dimensional manifold $M^{n}$ which has no triple points. Note that when $n>2$, the latter condition is automatically satisfied. The self-intersection set $A(g)$ of $g$ is a closed 1-dimensional submanifold of $M^{n}$ and $g(A(g))$ is also a closed 1-dimensional submanifold of $\mathbf{R}^{2 n-1}$. We say that a component $C$ of $g(A(g))$ is non-trivial if the double cover $g \mid g^{-1}(C): g^{-1}(C) \rightarrow C$ is non-trivial, i.e., if $g^{-1}(C)$ is connected. Let the number of non-trivial components be denoted by $\delta(g)$. In [19] Szücs has proved the following.

Theorem 1.1 ([19]). (1) Suppose that $f: M^{n} \rightarrow \mathbf{R}^{2 n}$ is an immersion with normal crossings and that $\pi: \mathbf{R}^{2 n} \rightarrow \mathbf{R}^{2 n-1}$ is a projection such that $g=\pi \circ f$ is an immersion with normal crossings without triple points. Then we have

$$
D(f) \equiv \delta(g) \quad(\bmod 2),
$$

where $D(f)$ denotes the number of double points of $f$.

(2) Suppose that $g: M^{n} \rightarrow \mathbf{R}^{2 n-1}$ is an immersion with normal crossings without triple points. If $n$ is even, we have

$$
\delta(g) \equiv\left\langle\bar{w}_{1}\left(M^{n}\right) \cup \bar{w}_{n-1}\left(M^{n}\right),\left[M^{n}\right]_{2}\right\rangle \quad(\bmod 2),
$$

where $\bar{w}_{i}\left(M^{n}\right) \in H^{i}\left(M^{n} ; \mathbf{Z}_{2}\right)$ denotes the $i$-th dual Stiefel-Whitney class of $M^{n}$ and $\left[M^{n}\right]_{2} \in H_{n}\left(M^{n} ; \mathbf{Z}_{2}\right)$ is the fundamental class of $M^{n}$.

As an interesting consequence of the above theorem we have the following.

Received by the editors November 29, 1994.

1991 Mathematics Subject Classification. Primary 57R42; Secondary 57R45, 57R40.

Key words and phrases. Double point circle, Whitney umbrella, normal Euler number, generic projection.

The first author was partially supported by CNPq, Brazil.

(C)1996 American Mathematical Society 
Corollary 1.2. Let $M^{n}$ be a closed $n$-dimensional manifold with $n$ even and $\bar{w}_{1}\left(M^{n}\right) \cup \bar{w}_{n-1}\left(M^{n}\right) \neq 0$. Then no immersion with normal crossings $g: M^{n} \rightarrow$ $\mathbf{R}^{2 n-1}$ without triple points can be lifted to an embedding into $\mathbf{R}^{2 n}$; i.e., there exist no projection $\pi: \mathbf{R}^{2 n} \rightarrow \mathbf{R}^{2 n-1}$ and no embedding $f: M^{n} \rightarrow \mathbf{R}^{2 n}$ with $g=\pi \circ f$.

The above corollary shows that $\delta(g)$ is an obstruction to the existence of a lift $f: M^{n} \rightarrow \mathbf{R}^{2 n}$ which is an embedding.

Suppose that we are given an immersion with normal crossings $f: M^{n} \rightarrow \mathbf{R}^{2 n}$. Then, in general, there does not exist a projection $\pi: \mathbf{R}^{2 n} \rightarrow \mathbf{R}^{2 n-1}$ such that $g=\pi \circ f: M^{n} \rightarrow \mathbf{R}^{2 n-1}$ is an immersion with normal crossings. In this situation, Mather [15] has shown that for a generic projection $\pi$, the map $g=\pi \circ f$ is "generic" in the sense of Whitney [24]; i.e., $g$ is an immersion with normal crossings outside a finite set of points, where the singular set consists of so-called Whitney umbrella points $[\mathbf{2 2}, \mathbf{2 4}]$. Such a map is called a stable map $([\mathbf{7}])$. Note that when $n>2$, every stable map $g: M^{n} \rightarrow \mathbf{R}^{2 n-1}$ has no triple points. Note also that the set of the stable maps is open and dense in $C^{\infty}\left(M^{n}, \mathbf{R}^{2 n-1}\right)$ with respect to the $C^{\infty}$ topology. Our main purpose of the present paper is to generalize the results of Szücs (Theorem 1.1) concerning immersions with normal crossings to stable maps $g: M^{n} \rightarrow \mathbf{R}^{2 n-1}$.

Let $g: M^{n} \rightarrow \mathbf{R}^{2 n-1}$ be a stable map without triple points. Then the closure of the self-intersection set $A(g)$ is a closed 1-dimensional submanifold of $M^{n}$ and the closure $\overline{g(A(g))}$ of $g(A(g))$ is a finite disjoint union of circles and arcs, where the two boundary points of each arc component correspond to Whitney umbrella points of $g$. If $g=\pi \circ f$ and $\mathbf{R}^{2 n}$ is oriented, where $f: M^{n} \rightarrow \mathbf{R}^{2 n}$ is an immersion with normal crossings and $\pi: \mathbf{R}^{2 n} \rightarrow \mathbf{R}^{2 n-1}$ is a projection, then we can define a sign, +1 or -1 , for each Whitney umbrella point of $g$, using a method essentially the same as that by Carter and Saito [5]. Our main result of the present paper is the following generalization of Theorem 1.1.

Theorem 1.3. (1) Suppose that $f: M^{n} \rightarrow \mathbf{R}^{2 n}$ is an immersion with normal crossings and that $\pi: \mathbf{R}^{2 n} \rightarrow \mathbf{R}^{2 n-1}$ is a projection such that $g=\pi \circ f$ is a stable map without triple points. Then we have

$$
D(f) \equiv \delta(g)+\varepsilon(f) \quad(\bmod 2) .
$$

(2) Suppose that $g: M^{n} \rightarrow \mathbf{R}^{2 n-1}$ is a stable map without triple points. If $n$ is even, we have

$$
\delta(g)+\beta(g) \equiv\left\langle\bar{w}_{1}\left(M^{n}\right) \cup \bar{w}_{n-1}\left(M^{n}\right),\left[M^{n}\right]_{2}\right\rangle \quad(\bmod 2) .
$$

Here the two terms $\varepsilon(f)$ and $\beta(g)$ in Theorem 1.3 denote the numbers of arc components $J$ of $\overline{g(A(g))}$ with some respective prescribed properties, which are described by the signs of the Whitney umbrella points corresponding to $\partial J$ and the triviality of the normal bundle of $g^{-1}(J)$ in $M^{n}$. Precise definitions will be given in $\S 3$.

As an application of our generalization, we give a new geometric proof of the following congruence formula, which has been proved by Mahowald [12] and Lannes [10] by using methods of algebraic topology. 
Theorem 1.4 (Mahowald [12], Lannes [10]). Let $f: M^{n} \rightarrow \mathbf{R}^{2 n}$ be an immersion with normal crossings, where $M^{n}$ is an $n$-dimensional closed manifold and $n \geq 2$ is even. Then we have

$$
\frac{e(f)}{2}+D(f) \equiv\left\langle\bar{w}_{1}\left(M^{n}\right) \cup \bar{w}_{n-1}\left(M^{n}\right),\left[M^{n}\right]_{2}\right\rangle \quad(\bmod 2),
$$

where $e(f)$ is the (twisted) Euler number of the normal bundle of $f$.

Note that, in our situation, the Euler number $e(f)$ is always an even integer. The above theorem is a generalization of the celebrated Whitney congruence for $n=2$ ([21]). Recall that the proofs by Mahowald [12] and Lannes $[\mathbf{1 0}]$ are algebraic in nature. Recently Carter and Saito [5] have given a geometric proof of the Whitney congruence using projections extensively together with surgery techniques. Our proof of the above theorem is motivated by their work, but is different from theirs. Our method is to find a generic projection $\pi: \mathbf{R}^{2 n} \rightarrow \mathbf{R}^{2 n-1}$ and to count the number of connected components of the double point set of the stable map $\pi \circ f$ : $M^{n} \rightarrow \mathbf{R}^{2 n-1}$ with some prescribed property.

Let $g: M^{n} \rightarrow \mathbf{R}^{2 n-1}$ be a stable map without triple points. Then, using our techniques, we see that the possible values of the normal Euler number of an embedding $f: M^{n} \rightarrow \mathbf{R}^{2 n}$ which is a lift of $g$ (i.e., $g=\pi \circ f$ for some projection $\pi: \mathbf{R}^{2 n} \rightarrow \mathbf{R}^{2 n-1}$ ) are somewhat restricted as follows.

Theorem 1.5. Let $g: M^{n} \rightarrow \mathbf{R}^{2 n-1}$ be a stable map and $\pi: \mathbf{R}^{2 n} \rightarrow \mathbf{R}^{2 n-1}$ a projection. Suppose that $n \geq 2$ and that $g$ has no triple points.

(1) There always exists an immersion with normal crossings $f: M^{n} \rightarrow \mathbf{R}^{2 n}$ such that $g=\pi \circ f$.

(2) There exists an embedding $f: M^{n} \rightarrow \mathbf{R}^{2 n}$ such that $g=\pi \circ f$ if and only if $\delta(g)=0$, i.e., if and only if $g$ has no non-trivial double point circle.

(3) For an embedding $f: M^{n} \rightarrow \mathbf{R}^{2 n}$ such that $g=\pi \circ f$, we have

$$
e(f) \in\{-2 \beta(g),-2 \beta(g)+4,-2 \beta(g)+8, \cdots, 2 \beta(g)\} .
$$

Furthermore, if $\delta(g)=0$, there exists an embedding $f: M^{n} \rightarrow \mathbf{R}^{2 n}$ with $g=\pi \circ f$ and with prescribed Euler number of the normal bundle in the above set.

Note that Theorem 1.5 (1) can be regarded as the existence theorem of a desingularization of a given stable map $f: M^{n} \rightarrow \mathbf{R}^{2 n-1}$ in the sense of Blank and Curley $[\mathbf{4},(1)]$. Theorem 1.5 (1) holds also for maps with triple points. Note also that Theorem 1.5 (3) is closely related to the well-known Whitney conjecture which has been proved by Massey [14] (see also [9]). See Remark 6.1 in $\S 6$.

Furthermore, we prove an elimination theorem of Whitney umbrella points of opposite signs, which leads to the following.

Theorem 1.6. Let $M^{n}$ be a closed connected $n$-dimensional manifold and $f$ : $M^{n} \rightarrow \mathbf{R}^{2 n}$ an embedding, where $n$ is even. Then there exists an isotopy $f_{t}$ : $M^{n} \rightarrow \mathbf{R}^{2 n}(t \in[0,1])$ such that $f_{0}=f$, that $\pi \circ f_{1}$ is stable and that $\pi \circ f_{1}$ has exactly $|e(f)|$ Whitney umbrella points, where $\pi: \mathbf{R}^{2 n} \rightarrow \mathbf{R}^{2 n-1}$ is a projection.

Note that this is a direct generalization of the main result of [5] concerning embedded surfaces in $\mathbf{R}^{4}$ to higher dimensions.

As to the problem of lifting a map into $\mathbf{R}^{2 n-1}$ to an embedding into $\mathbf{R}^{2 n}$, we obtain the following refinement of Corollary 1.2. 
Theorem 1.7. Let $M^{n}$ be a closed $n$-dimensional manifold $(n \geq 2)$ and $\pi: \mathbf{R}^{2 n} \rightarrow$ $\mathbf{R}^{2 n-1}$ a projection. Then the following three are equivalent.

(1) No immersion $g: M^{n} \rightarrow \mathbf{R}^{2 n-1}$ can be lifted to an embedding into $\mathbf{R}^{2 n}$.

(2) For every embedding $f: M^{n} \rightarrow \mathbf{R}^{2 n}$, the map $\pi \circ f$ can never be an immersion.

(3) $\left\langle\bar{w}_{1}\left(M^{n}\right) \cup \bar{w}_{n-1}\left(M^{n}\right),\left[M^{n}\right]_{2}\right\rangle \not \equiv 0 \quad(\bmod 2)$.

We also obtain the following result concerning the lifting problem.

Theorem 1.8. Let $M^{n}$ be a closed $n$-dimensional manifold $(n \geq 3)$ and $\pi: \mathbf{R}^{2 n} \rightarrow$ $\mathbf{R}^{2 n-1}$ a projection.

(1) If $M^{n}$ is orientable and $n$ is even, then, for every stable map $g: M^{n} \rightarrow$ $\mathbf{R}^{2 n-1}$, there exists an embedding $f: M^{n} \rightarrow \mathbf{R}^{2 n}$ such that $\pi \circ f=g$.

(2) If $M^{n}$ is non-orientable or $n$ is odd, then there exists an immersion with normal crossings $g: M^{n} \rightarrow \mathbf{R}^{2 n-1}$ which can never be lifted to an embedding into $\mathbf{R}^{2 n}$.

The paper is organized as follows. In $\S 2$, we define the sign of each Whitney umbrella point of a generic projection of an immersed $n$-manifold in $\mathbf{R}^{2 n}$ and relate it to the normal Euler number of the immersed manifold. In $\S 3$, we give a precise definition of the quantities $\varepsilon(f)$ and $\beta(g)$ appearing in Theorem 1.3. $\S 4$ is devoted to the proof of Theorem 1.3. In $\S 5$ we prove Theorem 1.4, using Theorem 1.3. We also consider an application of the Mahowald-Lannes congruence to the study of fold singular points. In $\S 6$, we consider the problem of lifting a map into $\mathbf{R}^{2 n-1}$ to an embedding into $\mathbf{R}^{2 n}$ and prove Theorems 1.5 and 1.8. In $\S 7$, we prove an elimination theorem of Whitney umbrella points and as a direct consequence we prove Theorem 1.6. Using this elimination theorem, we also prove Theorem 1.7.

Throughout the paper, $M^{n}$ denotes a closed $n$-dimensional manifold, all manifolds and maps are of class $C^{\infty}$, and the homologies and the cohomologies are with $\mathbf{Z}_{2}$-coefficients unless otherwise indicated.

The authors would like to express their sincere gratitude to Masahico Saito for pointing out a mistake in an earlier version of this paper.

\section{Signs of Whitney umbrella POINTS AND NORMAl Euler number}

Let $f: M^{n} \rightarrow \mathbf{R}^{2 n}$ be an immersion with normal crossings; i.e., $f$ is an embedding except at a finite number of transverse double points. Our purpose of this section is to find a generic projection $\pi: \mathbf{R}^{2 n} \rightarrow \mathbf{R}^{2 n-1}$, to define the sign of each Whitney umbrella point of $\pi \circ f$ and to study the normal Euler number of $f$. Set $A(f)=\left\{x \in M^{n}: f^{-1}(f(x)) \neq\{x\}\right\}$, which is called the self-intersection set of $f$.

Let $g: M^{n} \rightarrow \mathbf{R}^{2 n-1}$ be a smooth map. We define the self-intersection set $A(g)$ of $g$ by $A(g)=\left\{x \in M^{n}: g^{-1}(g(x)) \neq\{x\}\right\}$. We say that $g$ is stable if the orbit of $g$ in the mapping space $C^{\infty}\left(M^{n}, \mathbf{R}^{2 n-1}\right)$ by the action of $\operatorname{Diff}\left(M^{n}\right) \times \operatorname{Diff}\left(\mathbf{R}^{2 n-1}\right)$ is an open set (see, for example, $[\mathbf{7}])$. It is known $([\mathbf{2 2}, \mathbf{2 4}])$ that $g$ is stable if and only if the singular set $S(g)=\left\{x \in M^{n}\right.$ : rank $\left.d g_{x}<n\right\}$ of $g$ is a discrete set, the self-intersection set $A(g)$ does not contain any singular point, $g \mid\left(M^{n}-S(g)\right)$ is an immersion with normal crossings, and every point $p$ in $S(g)$ is a Whitney umbrella point; i.e., there exist local coordinates $\left(x_{1}, \cdots, x_{n}\right)$ centered at $p$ and $\left(y_{1}, \cdots, y_{2 n-1}\right)$ centered at $g(p)$ such that $g$ has the following normal form:

$$
y_{1} \circ g=x_{1}^{2}, y_{i} \circ g=x_{i}(i=2, \cdots, n), y_{n+j} \circ g=x_{1} x_{j+1}(j=1, \cdots, n-1) .
$$




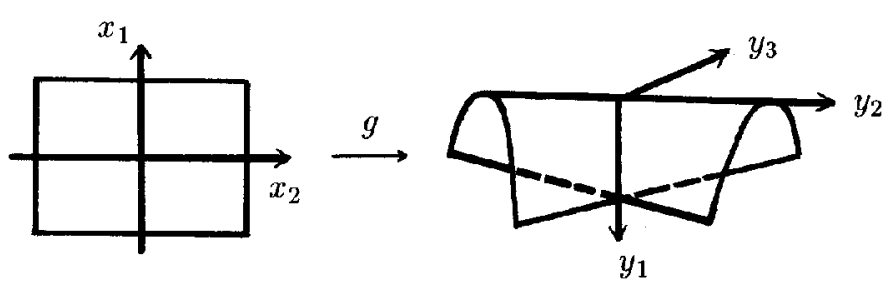

Figure 1. Whitney umbrella for the case $n=2$

(See Figure 1.) Note that, for every Whitney umbrella point $p$, rank $d g_{p}=n-1$.

Lemma 2.1. For almost every projection $\pi: \mathbf{R}^{2 n} \rightarrow \mathbf{R}^{2 n-1}$, the composite map $g=\pi \circ f: M^{n} \rightarrow \mathbf{R}^{2 n-1}$ is stable and non-singular at every point of $A(f)$.

Here we say that a linear map $\pi: \mathbf{R}^{k} \rightarrow \mathbf{R}^{l}(k>l)$ is a projection if it is surjective. A property is said to be true for almost every projection if the set of the projections for which the property is not true is of Lebesgue measure zero in the space of all projections.

Proof of Lemma 2.1. By Mather [15], for almost every projection $\pi: \mathbf{R}^{2 n} \rightarrow \mathbf{R}^{2 n-1}$, the composite $g=\pi \circ f$ is stable. On the other hand, the self-intersection set $A(f)$ consists of a finite number of points. For $x \in A(f)$, set $\Sigma_{x}$ to be the set of the projections $\pi: \mathbf{R}^{2 n} \rightarrow \mathbf{R}^{2 n-1}$ for which $\pi \circ f$ is singular at $x$. Since rank $d f_{x}=n$, the codimension of $\Sigma_{x}$ in the space of all projections is equal to $(2 n-1)-(n-1)=n \geq 1$. Hence $\bigcup_{x \in A(f)} \Sigma_{x}$ is of Lebesgue measure zero in the space of all projections. This completes the proof. $\|$

Let $\pi: \mathbf{R}^{2 n} \rightarrow \mathbf{R}^{2 n-1}$ be a projection as in Lemma 2.1. Take a Whitney umbrella point $p \in S(g)$, where $g=\pi \circ f$. We assume that $\mathbf{R}^{2 n}$ and $\mathbf{R}^{2 n-1}$ are oriented. We will define the sign of $p$ as follows.

Take local coordinates around $p$ and $g(p)$ as in the paragraph just before Lemma 2.1. Set $D_{\varepsilon}=\left\{y_{1}^{2}+\cdots+y_{2 n-1}^{2} \leq \varepsilon\right\} \subset \mathbf{R}^{2 n-1}$ for $\varepsilon>0$ sufficiently small. Set $\tilde{D}_{\varepsilon}=g^{-1}\left(D_{\varepsilon}\right)$.

Lemma 2.2. The set $\tilde{D}_{\varepsilon}$ is a closed disk neighborhood of $p$ in $M^{n}$.

Proof. It is easy to see that $\left(x_{1}, \cdots, x_{n}\right)$ is in $\tilde{D}_{\varepsilon}$ if and only if

$$
x_{2}^{2}+\cdots+x_{n}^{2} \leq \frac{\varepsilon^{2}-x_{1}^{4}}{1+x_{1}^{2}} .
$$

Then the result follows immediately. This completes the proof. ॥

Note that we have also shown that $\partial \tilde{D}_{\varepsilon}=g^{-1}\left(\partial D_{\varepsilon}\right)$.

In the following, we denote by $O_{X}$ the orientation of a space $X$. First we orient $\operatorname{ker} \pi$ so that $O_{\mathbf{R}^{2 n}}=O_{\mathbf{R}^{2 n-1}} \oplus O_{\operatorname{ker} \pi}$. It is easy to see that, if $\varepsilon$ is sufficiently small, $A(g) \cap \partial \tilde{D}_{\varepsilon}$ consists of two points. Let these two points be denoted by $p_{1}$ and $p_{2}$. Then the vector $f\left(p_{1}\right)-f\left(p_{2}\right)$ is an element of $\operatorname{ker} \pi$ and is not the zero vector, since $A(f)$ does not contain any Whitney umbrella point of $g$. We may assume that $f\left(p_{1}\right)-f\left(p_{2}\right)$ is consistent with the orientation of ker $\pi$, exchanging $p_{1}$ and $p_{2}$ if necessary. We fix an orientation of $\partial \tilde{D}_{\varepsilon}$ and take an oriented base $\left(u_{1}, \cdots, u_{n-1}\right)$ 
(resp. $\left.\left(v_{1}, \cdots, v_{n-1}\right)\right)$ of the tangent space $T_{p_{1}}\left(\partial \tilde{D}_{\varepsilon}\right)\left(\right.$ resp. $\left.T_{p_{2}}\left(\partial \tilde{D}_{\varepsilon}\right)\right)$. Then we see that $\left(d g_{p_{1}}\left(u_{1}\right), \cdots, d g_{p_{1}}\left(u_{n-1}\right), d g_{p_{2}}\left(v_{1}\right), \cdots, d g_{p_{2}}\left(v_{n-1}\right)\right)$ is a base of $T_{q}\left(\partial D_{\varepsilon}\right)$, where $q=g\left(p_{1}\right)=g\left(p_{2}\right)$. We denote this orientation by $O_{q}$. Recall that $D_{\varepsilon}$ is oriented, and hence its boundary $\partial D_{\varepsilon}$ is also oriented. Here we adopt the convention that $O_{\partial D_{\varepsilon}} \oplus \xi=O_{D_{\varepsilon}}$, where $\xi$ is the outward normal vector of $\partial D_{\varepsilon}$.

Definition 2.3. We say that the Whitney umbrella point $p \in S(\pi \circ f)$ is positive if $O_{q}=O_{\partial D_{\varepsilon}}$ and negative otherwise. We denote by $p(f)$ (resp. $n(f)$ ) the number of positive (resp. negative) Whitney umbrella points.

Remark 2.4. (1) The above definition does not depend on the choice of the orientation of $\partial \tilde{D}_{\varepsilon}$. It only depends on the orientations of $\mathbf{R}^{2 n}$ and $\mathbf{R}^{2 n-1}$.

(2) When $n$ is even, the above definition does not depend on the choice of the orientation of $\mathbf{R}^{2 n-1}$.

(3) When $n=2$, the above definition coincides with the definition due to Banchoff [3] and Carter and Saito [5].

Proposition 2.5. Suppose that $n$ is even and that $\mathbf{R}^{2 n}$ is oriented. Then we always have

$$
e(f)=p(f)-n(f),
$$

where $e(f) \in \mathbf{Z}$ is the (twisted) Euler number of the normal bundle of the immersion $f$.

Note that, when $n=2$ and $f$ is an embedding, this is a result of Banchoff $[\mathbf{3}$, $\S 4]$ and Carter and Saito [5].

Proof of Proposition 2.5. First note that the Euler number of the normal bundle of $f$ is equal to the intersection number of $f\left(M^{n}\right)$ and its parallel copy obtained by pushing $f\left(M^{n}\right)$ off itself slightly to the positive direction of ker $\pi$, where we ignore the intersections corresponding to the self-intersection points of $f\left(M^{n}\right)$. Since $g=\pi \circ f$ is an immersion off the Whitney umbrella points, we have only to investigate the contribution of each Whitney umbrella point to the intersection number. Let $p \in M^{n}$ be a Whitney umbrella point and set $q=f(p)$. Orient the disk neighborhood $\tilde{D}_{\varepsilon}$ of $p$ arbitrarily. Then the contribution of the Whitney umbrella point $p$ to the intersection number is equal to the intersection number of $f\left(\tilde{D}_{\varepsilon}\right)$ and its parallel copy $D^{\prime}$. By our orientation convention for the boundary, it is not difficult to see that this is equal to the intersection number of $D^{\prime \prime}$ and $\partial D^{\prime}$ in $\pi^{-1}\left(\partial D_{\varepsilon}\right)$, where $D^{\prime \prime}$ is a canonical $n$-disk in $\pi^{-1}\left(\partial D_{\varepsilon}\right)$ bounded by $f\left(\tilde{D}_{\varepsilon}\right) \cap \pi^{-1}\left(\partial D_{\varepsilon}\right)=f\left(\partial \tilde{D}_{\varepsilon}\right)$ and it intersects $\partial D^{\prime}$ transversely in one point (see Figure 2). (Note that $\pi^{-1}\left(\partial D_{\varepsilon}\right)$ is oriented as the boundary of $\pi^{-1}\left(D_{\varepsilon}\right)$.) Furthermore, this intersection number is easily seen to be equal to the sign of $\left(d f_{p_{1}}\left(u_{1}\right), \cdots\right.$, $\left.d f_{p_{1}}\left(u_{n-1}\right), f\left(p_{1}\right)-f\left(p_{2}\right), d f_{p_{2}}\left(v_{1}\right), \cdots, d f_{p_{2}}\left(v_{n-1}\right)\right)$ in $\pi^{-1}\left(\partial D_{\varepsilon}\right)$, where $p_{1}, p_{2} \in$ $M^{n}, u_{1}, \cdots, u_{n-1} \in T_{p_{1}}\left(\partial \tilde{D}_{\varepsilon}\right)$ and $v_{1}, \cdots, v_{n-1} \in T_{p_{2}}\left(\partial \tilde{D}_{\varepsilon}\right)$ are as in the paragraph just before Definition 2.3. Then this is equal to the sign of $\left(d f_{p_{1}}\left(u_{1}\right), \cdots\right.$, $\left.d f_{p_{1}}\left(u_{n-1}\right), f\left(p_{1}\right)-f\left(p_{2}\right), d f_{p_{2}}\left(v_{1}\right), \cdots, d f_{p_{2}}\left(v_{n-1}\right), \xi\right)$ in $\mathbf{R}^{2 n}$, where $\xi$ is the outward normal vector of $\partial\left(\pi^{-1}\left(D_{\varepsilon}\right)\right)$. Since $n$ is even, we see that this is equal to the sign of $\left(d f_{p_{1}}\left(u_{1}\right), \cdots, d f_{p_{1}}\left(u_{n-1}\right), d f_{p_{2}}\left(v_{1}\right), \cdots, d f_{p_{2}}\left(v_{n-1}\right), \xi, f\left(p_{1}\right)-f\left(p_{2}\right)\right)$, which is equal, by the orientation convention of ker $\pi$, to the sign of $\left(d g_{p_{1}}\left(u_{1}\right), \cdots, d g_{p_{1}}\left(u_{n-1}\right)\right.$, $\left.d g_{p_{2}}\left(v_{1}\right), \cdots, d g_{p_{2}}\left(v_{n-1}\right), \xi^{\prime}\right)$ in $\mathbf{R}^{2 n-1}$, where $\xi^{\prime}$ is the outward normal of $\partial D_{\varepsilon}$. Then, by the orientation convention of the boundary, this is equal to the sign of 


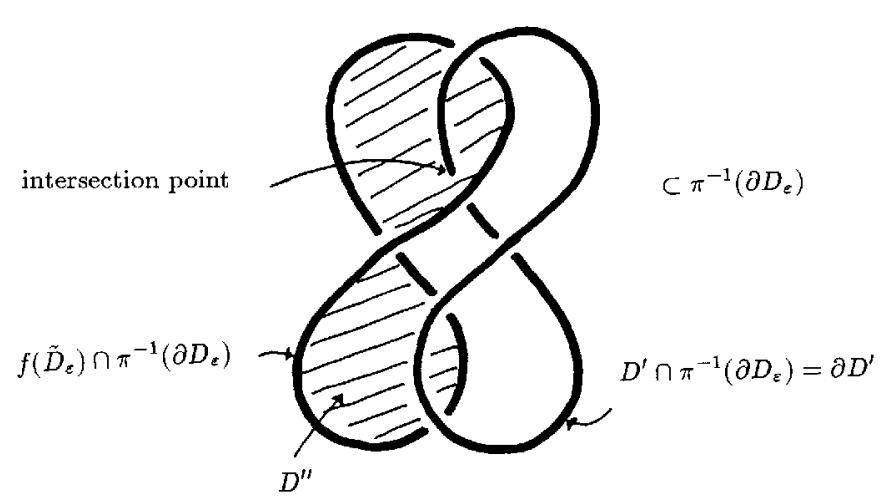

FiguRE 2

$\left(d g_{p_{1}}\left(u_{1}\right), \cdots, d g_{p_{2}}\left(u_{n-1}\right), d g_{p_{2}}\left(v_{1}\right), \cdots, d g_{p_{2}}\left(v_{n-1}\right)\right)$ in $\partial D_{\varepsilon}$. Hence, this is equal to +1 if $p$ is a positive Whitney umbrella point and -1 if it is a negative one. This completes the proof. II

\section{NON-TRIVIAL CIRCLES AND ARCS}

Let $f: M^{n} \rightarrow \mathbf{R}^{2 n}$ be an immersion with normal crossings. In what follows, we assume that $\mathbf{R}^{2 n}$ is oriented. Take a projection $\pi: \mathbf{R}^{2 n} \rightarrow \mathbf{R}^{2 n-1}$ as in Lemma 2.1 and set $g=\pi \circ f$, which is a stable map. We assume that, when $n=2, g$ has no triple points. (For the case where $g$ has triple points, see the last paragraph of the proof of Theorem 1.4 and Remark 5.1 in $\S 5$.) Set $B(g)=g(A(g))$. It is easy to see that the closure $\overline{A(g)}$ of $A(g)$ in $M^{n}$ is a closed 1-dimensional submanifold of $M^{n}$ without boundary. Furthermore, the closure $\overline{B(g)}$ of $B(g)$ in $\mathbf{R}^{2 n-1}$ is a compact 1-dimensional submanifold of $\mathbf{R}^{2 n-1}$ with boundary corresponding to the Whitney umbrella points of $g$. Note that, if $J$ is an arc component of $\overline{B(g)}$, then $\tilde{J}=g^{-1}(J)$ is a smooth submanifold of $M^{n}$ diffeomorphic to $S^{1}$.

Definition 3.1. A circle component $C$ of $\overline{B(g)}$ is said to be non-trivial if $g^{-1}(C)$ is connected. An arc component $J$ of $\overline{B(g)}$ is said to be non-trivial if either of the following two conditions is satisfied:

(1) the normal bundle of $\tilde{J}$ in $M^{n}$ is trivial and the signs of the two Whitney umbrella points $\partial J$ coincide, or

(2) the normal bundle of $\tilde{J}$ in $M^{n}$ is non-trivial and the signs of the two Whitney umbrella points $\partial J$ do not coincide.

Here we note that the above definition does not depend on the orientation of $\mathbf{R}^{2 n-1}$ even when $n$ is odd. We denote by $\delta(g)$ the number of non-trivial circle components, by $\varepsilon(f)$ the number of non-trivial arc components, by $\alpha(f)$ the number of arc components $J$ such that the signs of the two Whitney umbrella points $\partial J$ coincide, and by $\beta(g)$ the number of arc components $J$ such that the normal bundle of $\tilde{J}$ in $M^{n}$ is non-trivial. Note that $\delta$ and $\beta$ depend only on $g$, while $\varepsilon$ and $\alpha$ depend on $f$ and the projection $\pi$.

We have the following lemma.

Lemma 3.2. $\alpha(f) \equiv \beta(g)+\varepsilon(f) \quad(\bmod 2)$. 
Proof. We denote by $a$ the number of trivial arc components $J$ with the same sign of the two Whitney umbrella points $\partial J$, by $b$ the number of trivial arc components $J$ with distinct signs of the Whitney umbrella points, by $c$ the number of non-trivial arc components with the same sign of the Whitney umbrella points, and by $d$ the number of non-trivial arc components with distinct signs of the Whitney umbrella points. Then we have $\alpha(f)=a+c, \beta(g)=c+d$, and $\varepsilon(f)=a+d$. Then the required congruence follows immediately. \|

\section{Proof of Theorem 1.3}

First we prove part (1) of Theorem 1.3. By Szücs [19], the number of double points of $f$ over the circle components of $\overline{B(g)}$ has the same parity as $\delta(g)$. Thus it suffices to prove that the number of double points of $f$ over the arc components of $\overline{B(g)}$ has the same parity as $\varepsilon(f)$. Hence, we have only to show the following.

Lemma 4.1. Let $J$ be an arc component of $\overline{B(g)}$. Then the number of double points of $f$ in $\pi^{-1}(J)$ is odd if and only if $J$ is non-trivial.

Proof. We denote by $q_{1}$ and $q_{2}$ the two end points of $J$ and set $p_{i}=g^{-1}\left(q_{i}\right)$ $(i=1,2)$. Let $D_{1}$ and $D_{2}$ be sufficiently small closed disk neighborhoods of $q_{1}$ and $q_{2}$ in $\mathbf{R}^{2 n-1}$ respectively as in $\S 2$. We may assume $D_{1} \cap D_{2}=\emptyset$. We may also assume that there are no double points of $f$ in $\pi^{-1}\left(J \cap D_{i}\right)(i=1,2)$. We denote by $J_{1}$ and $J_{2}$ the two components of the closure of $g^{-1}\left(J-\left(D_{1} \cup D_{2}\right)\right)$. Set $g^{-1}\left(D_{i}\right) \cap J_{j}=\left\{p_{i j}\right\}$ $(i, j=1,2)$ (see Figure 3). We fix orientations of $\tilde{D}_{1}=g^{-1}\left(D_{1}\right)$ and $\mathbf{R}^{2 n-1}$. We may assume that $f\left(p_{11}\right)-f\left(p_{12}\right)$ gives the orientation of $\operatorname{ker} \pi$, exchanging $p_{11}$ and $p_{12}$ if necessary. Let $\left(u_{1}, \cdots, u_{n-1}\right)$ (resp. $\left.\left(v_{1}, \cdots, v_{n-1}\right)\right)$ be an oriented base of $T_{p_{11}}\left(\partial \tilde{D}_{1}\right)$ (resp. $\left.T_{p_{12}}\left(\partial \tilde{D}_{1}\right)\right)$. This is also regarded as a base of a fiber over

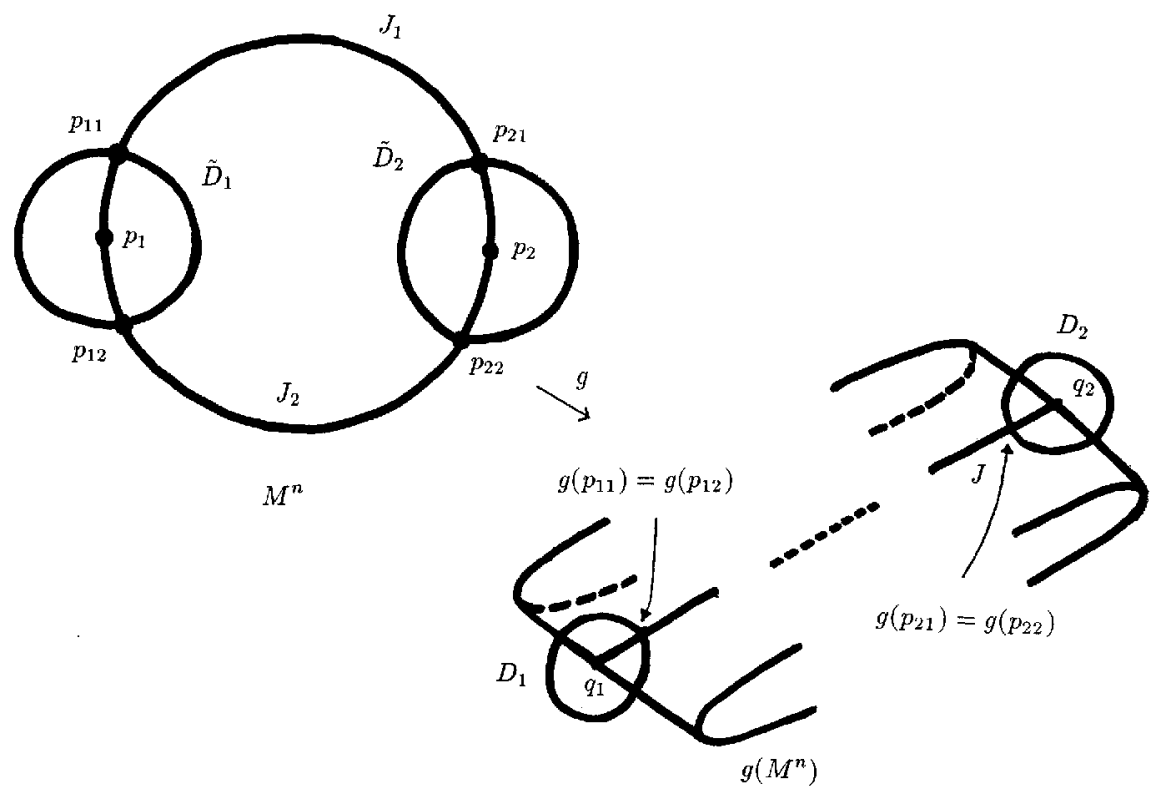

FiguRE 3 
$p_{11}$ (resp. $p_{12}$ ) of the normal bundle of $J_{1}$ (resp. $J_{2}$ ) in $M^{n}$. Since the normal bundle of $J_{j}$ is trivial, the above base can be extended to a trivialization $\left(U_{1}, \cdots, U_{n-1}\right)$ (resp. $\left.\left(V_{1}, \cdots, V_{n-1}\right)\right)$ of the normal bundle. Let $\left(t_{1}, \cdots, t_{n-1}\right)$ (resp. $\left.\left(w_{1}, \cdots, w_{n-1}\right)\right)$ denote the base of the fiber over $p_{21}$ (resp. $p_{22}$ ) of the normal bundle of $J_{1}$ (resp. $J_{2}$ ) which corresponds to the extended trivialization $\left(U_{1}, \cdots, U_{n-1}\right)\left(\right.$ resp. $\left.\left(V_{1}, \cdots, V_{n-1}\right)\right)$. Note that $\left(t_{1}, \cdots, t_{n-1}\right)$ and $\left(w_{1}, \cdots, w_{n-1}\right)$ are regarded as bases of $T_{p_{21}}\left(\partial \tilde{D}_{2}\right)$ and $T_{p_{22}}\left(\partial \tilde{D}_{2}\right)$ respectively. Then we see that $\left(d g_{p_{11}}\left(u_{1}\right), \cdots, d g_{p_{11}}\left(u_{n-1}\right), d g_{p_{12}}\left(v_{1}\right), \cdots, d g_{p_{12}}\left(v_{n-1}\right)\right)$ gives the orientation of $\partial D_{1}$ if and only if $\left(d g_{p_{21}}\left(t_{1}\right), \cdots, d g_{p_{21}}\left(t_{n-1}\right), d g_{p_{22}}\left(w_{1}\right), \cdots, d g_{p_{22}}\left(w_{n-1}\right)\right)$ does not give the orientation of $\partial D_{2}$, since $\left(d g\left(U_{1}\right), \cdots, d g\left(U_{n-1}\right), d g\left(V_{1}\right), \cdots, d g\left(V_{n-1}\right)\right)$ is a trivialization of the normal bundle of the closure of $J-\left(D_{1} \cup D_{2}\right)$ in $\mathbf{R}^{2 n-1}$. Moreover, it is easy to see that $f\left(p_{21}\right)-f\left(p_{22}\right)$ is not consistent with the orientation of $\operatorname{ker} \pi$ if and only if the number of double points of $f$ in $\pi^{-1}(J)$ is odd. Now we orient $\tilde{D}_{2}=g^{-1}\left(D_{2}\right)$ so that $\left(t_{1}, \cdots, t_{n-1}\right)$ is an oriented base of $T_{p_{21}}\left(\partial \tilde{D}_{2}\right)$. Note that $\left(w_{1}, \cdots, w_{n-1}\right)$ is consistent with the orientation of $T_{p_{22}}\left(\partial \tilde{D}_{2}\right)$ if and only if the normal bundle of $\tilde{J}=g^{-1}(J)$ in $M^{n}$ is trivial.

Now suppose that $p_{2}$ is a positive Whitney umbrella point and that the normal bundle of $\tilde{J}$ is trivial. Then the number of double points of $f$ in $\pi^{-1}(J)$ is even if and only if $f\left(p_{21}\right)-f\left(p_{22}\right)$ is consistent with the orientation of ker $\pi$, which is true if and only if $\left(d g_{p_{21}}\left(t_{1}\right), \cdots, d g_{p_{21}}\left(t_{n-1}\right), d g_{p_{22}}\left(w_{1}\right), \cdots, d g_{p_{22}}\left(w_{n-1}\right)\right)$ is consistent with the orientation of $\partial D_{2}$. This is true if and only if $\left(d g_{p_{11}}\left(u_{1}\right), \cdots, d g_{p_{11}}\left(u_{n-1}\right)\right.$, $\left.d g_{p_{12}}\left(v_{1}\right), \cdots, d g_{p_{12}}\left(v_{n-1}\right)\right)$ is not consistent with the orientation of $\partial D_{1}$, which is true if and only if $p_{1}$ is a negative Whitney umbrella point. This is true if and only if the arc component $J$ is trivial.

The other cases where $p_{2}$ is a negative Whitney umbrella point or the normal bundle of $\tilde{J}$ is non-trivial are treated similarly. This completes the proof of Lemma 4.1 and hence Theorem 1.3 (1). ॥

Now we prove part (2) of Theorem 1.3. Let $g: M^{n} \rightarrow \mathbf{R}^{2 n-1}$ be a stable map without triple points with $n$ even. First we give a proof based on results of Szücs [19] which works for $n>2$. Take an $\operatorname{arc}$ component $J$ of $\overline{B(g)}$. Then we can eliminate the two Whitney umbrella points $\partial J$ by a surgery technique similar to that used in [18]. (The technique is considered only for $n=2$ in [18], but the same idea is valid also for general $n$.) More precisely, we remove small ball neighborhoods of $g^{-1}(\partial J)$ and replace it with $S^{n-1} \times[-1,1]$. Constructing an immersion into $\mathbf{R}^{2 n-1}$ properly on this cylinder, we can reduce the number of Whitney umbrella points of $g$ by two, preserving the property that $g=\pi \circ f$ has no triple point, since $n>2$. Note that, by this procedure, the arc component $J$ turns into a circle component. In this way, we may assume that $g$ has no Whitney umbrella points. Furthermore, it is not difficult to see that the normal bundle of $g^{-1}(J)$ in $M^{n}$ is trivial if and only if the new circle component corresponding to $J$ is trivial. Then Theorem 1.3 (2) follows from Theorem 1.1 (2). Note that the parity of

$$
\left\langle\bar{w}_{1}\left(M^{n}\right) \cup \bar{w}_{n-1}\left(M^{n}\right),\left[M^{n}\right]_{2}\right\rangle
$$

does not change by the surgery operation as above, since this is a cobordism invariant.

Unfortunately, the above argument works only for $n>2$. In the following, we give a proof which works for all $n \geq 2$ and which is elementary in nature. 


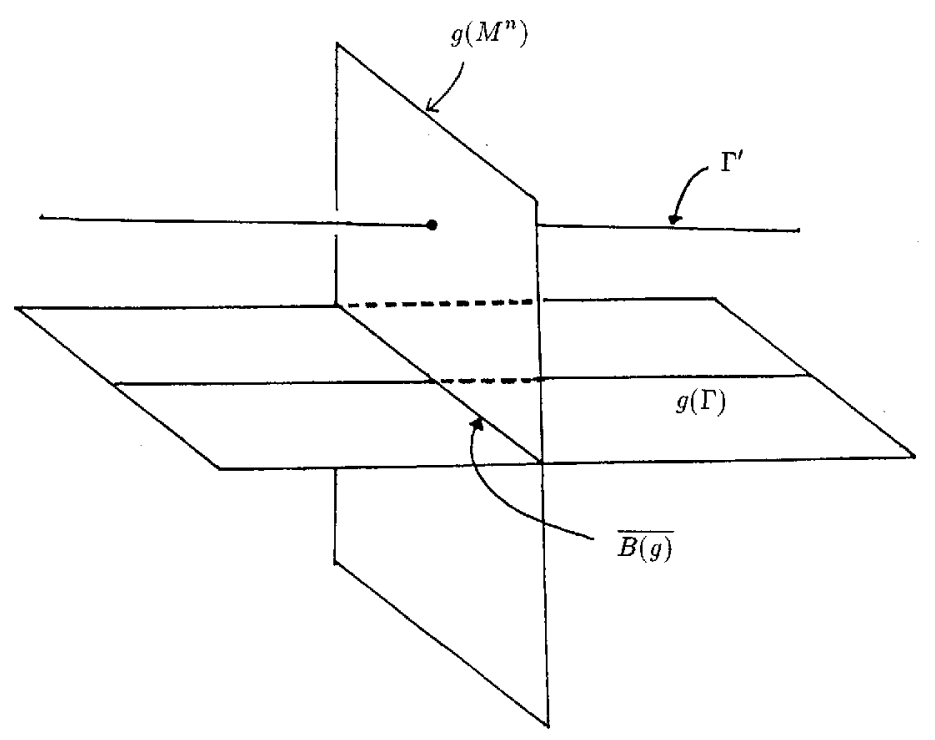

FiguRe 4

Recall that the set $S=S(g)$ of the Whitney umbrella points of $g$ is a finite set. Take a small tubular neighborhood $N(S)$ of $S$ in $M^{n}$. Note that $g \mid\left(M^{n}-N(S)\right)$ is an immersion, whose normal bundle is denoted by $\nu_{1}$.

Lemma 4.2. We have

$$
i^{*} \bar{w}_{n-1}\left(M^{n}\right)=w_{n-1}\left(\nu_{1}\right),
$$

where $i: M^{n}-N(S) \rightarrow M^{n}$ is the inclusion map.

Proof. Since $\nu_{1}$ is a normal bundle of $M^{n}-N(S), w_{j}\left(\nu_{1}\right)$ is a polynomial in $w_{k}\left(M^{n}-N(S)\right)=i^{*} w_{k}\left(M^{n}\right)$. On the other hand, $\bar{w}_{j}\left(M^{n}\right)$ is a polynomial in $w_{k}\left(M^{n}\right)$ with the same form. Then we see easily that $i^{*} \bar{w}_{j}\left(M^{n}\right)=w_{j}\left(\nu_{1}\right)$. This completes the proof. $\|$

Lemma 4.3. We have $[\overline{A(g)}]_{2}^{*}=\bar{w}_{n-1}\left(M^{n}\right)$ in $H^{n-1}\left(M^{n}\right)$, where $[\overline{A(g)}]_{2}^{*} \in$ $H^{n-1}\left(M^{n}\right)$ is the Poincaré dual of the homology class $[\overline{A(g)}]_{2} \in H_{1}\left(M^{n}\right)$ represented by the closure of $A(g)$.

Proof. By Poincaré duality, we have only to show that

$$
[\overline{A(g)}]_{2} \cdot \gamma=\left\langle\bar{w}_{n-1}\left(M^{n}\right), \gamma\right\rangle
$$

for every $\gamma \in H_{n-1}\left(M^{n}\right)$, where $[\overline{A(g)}]_{2} \cdot \gamma$ is the $\mathbf{Z}_{2}$-intersection number. Let $\Gamma$ be a singular $(n-1)$-chain in $M^{n}$ representing $\gamma$. (In fact, by Thom [20], there exists a closed $(n-1)$-dimensional submanifold $V$ of $M^{n}$ which represents $\gamma \in H_{n-1}\left(M^{n}\right)$. However, we do not need this here.) By a transversality argument, we may assume that $\Gamma \cap N(S)=\emptyset$, that $\Gamma$ intersects $\overline{A(g)}$ transversely in a finite number of points. Then we have

$$
[\overline{A(g)}]_{2} \cdot \gamma \equiv \sharp(\Gamma \cap \overline{A(g)}) \quad(\bmod 2),
$$


where $\sharp$ denotes the number of elements in the set. Let $\Gamma^{\prime}$ be a singular $(n-1)$-chain in $\mathbf{R}^{2 n-1}$ which is obtained by a small homotopy of $g(\Gamma)$ so that $\Gamma^{\prime} \cap \overline{B(g)}=\emptyset$, that $\Gamma^{\prime}$ intersects $g\left(M^{n}\right)$ transversely in a finite number of points, and that $\Gamma^{\prime}$ and $g\left(M^{n}\right)$ intersect in one point in a neighborhood of each point of $g(\Gamma \cap \overline{A(g)}$ ) (see Figure 4). Since $H_{n-1}\left(\mathbf{R}^{2 n-1}\right) \cong H_{n}\left(\mathbf{R}^{2 n-1}\right)=0$, we have

$$
\sharp\left(\Gamma^{\prime} \cap g\left(M^{n}\right)\right) \equiv 0 \quad(\bmod 2) .
$$

On the other hand, by Lemma 4.2, we have

$$
\begin{aligned}
\left\langle w_{n-1}\left(\nu_{1}\right),[\Gamma]_{2}\right\rangle & =\left\langle i^{*} \bar{w}_{n-1}\left(M^{n}\right),[\Gamma]_{2}\right\rangle \\
& =\left\langle\bar{w}_{n-1}\left(M^{n}\right), \gamma\right\rangle,
\end{aligned}
$$

where $[\Gamma]_{2}=\gamma$ is the homology class represented by $\Gamma$. Furthermore, we have

$$
\sharp\left(\Gamma^{\prime} \cap g\left(M^{n}\right)\right) \equiv\left\langle w_{n-1}\left(\nu_{1}\right),[\Gamma]_{2}\right\rangle+\sharp(\Gamma \cap \overline{A(g)}) \quad(\bmod 2),
$$

since $\left\langle w_{n-1}\left(\nu_{1}\right),[\Gamma]_{2}\right\rangle$ is congruent modulo 2 to the number of intersection points of $\Gamma^{\prime}$ and $g\left(M^{n}\right)$ off the neighborhood of $g(\Gamma \cap \overline{A(g)})$. Then, by (4.5), (4.6) and (4.7), we have

$$
\sharp(\Gamma \cap \overline{A(g)}) \equiv\left\langle\bar{w}_{n-1}\left(M^{n}\right), \gamma\right\rangle \quad(\bmod 2) .
$$

Combining this with (4.4), we have

$$
[\overline{A(g)}]_{2} \cdot \gamma \equiv\left\langle\bar{w}_{n-1}\left(M^{n}\right), \gamma\right\rangle \quad(\bmod 2) .
$$

This completes the proof of Lemma 4.3. I

Here we note that Lemma 4.3 can also be proved using Ronga's result [16].

Let $\nu$ denote the normal bundle of $\overline{A(g)}$ in $M^{n}$ and $\varepsilon^{1}$ the trivial line bundle over $\overline{A(g)}$. Then we see that $\nu \oplus \varepsilon^{1}$ is isomorphic to $T M^{n} \mid \overline{A(g)}$. Hence we have

$$
w_{1}(\nu)=w_{1}\left(T M^{n} \mid \overline{A(g)}\right) .
$$

Thus we have

$$
\begin{aligned}
\left\langle w_{1}(\nu),[\overline{A(g)}]_{2}\right\rangle & =\left\langle w_{1}\left(T M^{n} \mid \overline{A(g)}\right),[\overline{A(g)}]_{2}\right\rangle \\
& =\left\langle w_{1}\left(M^{n}\right),[\overline{A(g)}]_{2}\right\rangle \\
& =\left\langle w_{1}\left(M^{n}\right),\left(\bar{w}_{n-1}\left(M^{n}\right)\right)^{*}\right\rangle \quad(\text { by Lemma 4.3) } \\
& =\left\langle\bar{w}_{1}\left(M^{n}\right) \cup \bar{w}_{n-1}\left(M^{n}\right),\left[M^{n}\right]_{2}\right\rangle,
\end{aligned}
$$

since $w_{1}\left(M^{n}\right)=\bar{w}_{1}\left(M^{n}\right)$, where $\left(\bar{w}_{n-1}\left(M^{n}\right)\right)^{*} \in H_{1}\left(M^{n}\right)$ is the Poincaré dual of $\bar{w}_{n-1}\left(M^{n}\right)$.

Lemma 4.9. Let $C$ be a circle component of $\overline{B(g)}$.

(1) If $C$ is non-trivial and $n$ is even, the normal bundle of $g^{-1}(C)$ in $M^{n}$ is always non-trivial. 
(2) If $C$ is trivial, we set $g^{-1}(C)=C_{1} \cup C_{2}$, where $C_{i}$ are the components. Then the normal bundle of $C_{1}$ in $M^{n}$ is trivial if and only if the normal bundle of $C_{2}$ in $M^{n}$ is trivial.

Proof. First we prove part (1). Suppose that the normal bundle $\nu_{0}$ of $g^{-1}(C)$ in $M^{n}$ is trivial. Fix a trivialization $\xi$ of $\nu_{0}$. Recall that $g \mid g^{-1}(C): g^{-1}(C) \rightarrow C$ is a double cover. Then we see that $d g(\xi)$ gives a trivialization of $\varphi^{*} \nu_{0}^{\prime}$, where $\nu_{0}^{\prime}$ is the normal bundle of $C$ in $\mathbf{R}^{2 n-1}$ and $\varphi:[0,1] \rightarrow C$ is a parametrization. Then the monodromy matrix of $\nu_{0}^{\prime}$ with respect to the trivialization of $\varphi^{*} \nu_{0}^{\prime}$ is of the form

$$
R=\left[\begin{array}{cc}
0 & A \\
B & 0
\end{array}\right]
$$

where $A$ and $B$ are $(n-1) \times(n-1)$ real matrices with $A B$ the identity matrix. Then we have

$$
\operatorname{det} R=(-1)^{(n-1)^{2}}(\operatorname{det} A) \cdot(\operatorname{det} B)=-1 .
$$

This contradicts the fact that $\nu_{0}^{\prime}$ is orientable. Thus $\nu_{0}$ must be non-trivial.

Now we prove part (2). Let $\nu_{i}^{\prime}$ denote the normal bundle of $C_{i}$ in $M^{n}(i=1,2)$. We regard $\nu_{i}^{\prime}$ as a bundle over $C$ by the diffeomorphism $g \mid C_{i}: C_{i} \rightarrow C$. Then we see that the bundle $\nu_{1}^{\prime} \oplus \nu_{2}^{\prime}$ is isomorphic to the normal bundle of $C$ in $\mathbf{R}^{2 n-1}$. Thus $\nu_{1}^{\prime} \oplus \nu_{2}^{\prime}$ is trivial and we have $w_{1}\left(\nu_{1}^{\prime}\right)=w_{1}\left(\nu_{2}^{\prime}\right)$. This completes the proof. $\|$

Now we go back to the proof of Theorem 1.3 (2). By Lemma 4.9 and the definition of $\beta(g)$, we have

$$
\left\langle w_{1}(\nu),\left[\overline{A(g)}_{2}\right\rangle \equiv \beta(g)+\delta(g) \quad(\bmod 2) .\right.
$$

This, together with (4.8), implies

$$
\left\langle\bar{w}_{1}\left(M^{n}\right) \cup \bar{w}_{n-1}\left(M^{n}\right),\left[M^{n}\right]_{2}\right\rangle \equiv \beta(g)+\delta(g) \quad(\bmod 2) .
$$

This completes the proof of Theorem $1.3(2)$. ॥

\section{Proof of the Mahowald-Lannes Congruence}

In this section we give a new geometric proof of the Mahowald-Lannes congruence formula as an application of our Theorem 1.3. We also consider an application of the congruence formula to the study of fold singular points.

Proof of Theorem 1.4. First we assume that $n>2$ and we continue to use the notations of the previous sections. We denote by $a_{+}$(resp. $a_{-}$) the number of arc components $J$ of $\overline{B(g)}$ such that the normal bundle of $\tilde{J}=g^{-1}(J)$ in $M^{n}$ is trivial and that the signs of the two Whitney umbrella points $\partial J$ are both positive (resp. negative). Furthermore, we denote by $c_{+}$(resp. $c_{-}$) the number of arc components $J$ such that the normal bundle of $\tilde{J}$ is non-trivial and that the signs of the two Whitney umbrella points $\partial J$ are both positive (resp. negative). We use the same 
notations as in the proof of Lemma 3.2. Then we have

$$
\begin{aligned}
\frac{e(f)}{2} & =\left(a_{+}+c_{+}\right)-\left(a_{-}+c_{-}\right) \quad(\text { by Proposition } 2.5) \\
& \equiv\left(a_{+}+a_{-}\right)+\left(c_{+}+c_{-}\right) \quad(\bmod 2) \\
& =a+c \\
& =\alpha(f) \\
& \equiv \beta(g)+\varepsilon(f) \quad(\bmod 2) \quad(\text { by Lemma } 3.2) \\
& \equiv \beta(g)+\delta(g)+D(f) \quad(\bmod 2) \quad(\text { by Theorem } 1.3(1)) \\
& \equiv\left\langle\bar{w}_{1}\left(M^{n}\right) \cup \bar{w}_{n-1}\left(M^{n}\right),\left[M^{n}\right]_{2}\right\rangle+D(f) \quad(\bmod 2)
\end{aligned}
$$

(by Theorem $1.3(2)$ ).

This completes the proof of Theorem 1.4 for $n>2$.

Now suppose that $n=2$. Then it is easy to see that there exists a projection $\pi: \mathbf{R}^{4} \rightarrow \mathbf{R}^{3}$ such that $g(A(f))$ does not contain any triple points of $g$. Then we can change $f$ by a surgery so that $g=\pi \circ f$ has no triple points, without changing the difference between the numbers of positive and negative Whitney umbrella points, the number of double points, and the parity of $\left\langle\bar{w}_{1}\left(M^{2}\right) \cup \bar{w}_{1}\left(M^{2}\right),\left[M^{2}\right]_{2}\right\rangle \equiv$ $\chi\left(M^{2}\right)(\bmod 2)$, where $\chi$ denotes the Euler characteristic. (For details, see [5, Figure N].) Hence, in order to prove Theorem 1.4, we may assume that $g$ has no triple points. Then the same argument as above can apply. This completes the proof. II

Remark 5.1. We have used surgery techniques in order to prove Theorem 1.4 for $n=2$. However, we can prove it without using surgery techniques, modifying the proof as follows. Suppose that $f: M^{2} \rightarrow \mathbf{R}^{4}$ is an immersion with normal crossings and that $\pi: \mathbf{R}^{4} \rightarrow \mathbf{R}^{3}$ is a projection as in Lemma 2.1 such that $g(A(f))$ contains no triple point, where $g=\pi \circ f$. Then there exist immersions with normal crossings $\alpha_{i}: S^{1} \rightarrow M^{2}(i=1, \cdots, r)$ such that $\bigcup_{i=1}^{r} \alpha_{i}\left(S^{1}\right)=\overline{A(g)}$ and that $\alpha_{i}\left(S^{1}\right)$ and $\alpha_{j}\left(S^{1}\right)$ intersect transversely if $i \neq j$. (In the terminology of Giller [6], each $\alpha_{i}\left(S^{1}\right)$ is a transverse component of $\overline{A(g)}$.) There are three types of $\alpha_{i}$ as follows.

(a) The set $\alpha_{i}\left(S^{1}\right)$ contains a Whitney umbrella point.

(b) The set $\alpha_{i}\left(S^{1}\right)$ does not contain a Whitney umbrella point and there exists one and only one $j(\neq i)$ such that $g\left(\alpha_{i}\left(S^{1}\right)\right)=g\left(\alpha_{j}\left(S^{1}\right)\right)$.

(c) The set $\alpha_{i}\left(S^{1}\right)$ does not contain a Whitney umbrella point and there does not exist $j(\neq i)$ such that $g\left(\alpha_{i}\left(S^{1}\right)\right)=g\left(\alpha_{j}\left(S^{1}\right)\right)$.

In the case of (a), it is easy to see that $\alpha_{i}\left(S^{1}\right)$ contains exactly two Whitney umbrella points. For $\alpha_{i}\left(S^{1}\right)$ containing no Whitney umbrella points, we say that it is non-trivial if it satisfies condition (c). For $\alpha_{i}\left(S^{1}\right)$ containing Whitney umbrella points, we say that it is non-trivial if the normal bundle of the immersion $\alpha_{i}$ is trivial and the signs of the two Whitney umbrella points contained in $\alpha_{i}\left(S^{1}\right)$ are the same, or if the normal bundle is non-trivial and the signs of the Whitney umbrella points are opposite to each other. (Compare this with Definition 3.1.) Furthermore, we define $\delta(g), \varepsilon(f), \alpha(f)$ and $\beta(g)$ as in $\S 3$. Then it is not difficult to see that Lemma 3.2 and Theorem 1.3 hold also for this case. Then we can prove Theorem 1.4 without using surgery techniques.

Remark 5.2. Theorem 1.4 was first proved by Mahowald $[\mathbf{1 2}]$ when $f$ is an embedding. Then Lannes [10] proved it for immersions with normal crossings in general. 
Note that Audin [1] gave another proof of Theorem 1.4 and generalized it for immersions with normal crossings of a closed $n$-dimensional manifold into a closed oriented $2 n$-dimensional manifold $[\mathbf{2}$, Lemme 2.5.2]. See also $[\mathbf{1 1}, \mathbf{2 5}]$.

Remark 5.3. Note that $\bar{w}_{1}\left(M^{n}\right) \cup \bar{w}_{n-1}\left(M^{n}\right)=0$ unless $n=2^{r}$ for some $r \geq$ 1 (see [13]). Conversely, for $n=2^{r}$, consider the real projective space $\mathbf{R} P^{n}$. Then it is known that $\left\langle\bar{w}_{1}\left(\mathbf{R} P^{n}\right) \cup \bar{w}_{n-1}\left(\mathbf{R} P^{n}\right),\left[\mathbf{R} P^{n}\right]_{2}\right\rangle \not \equiv 0$. Note also that $\left\langle\bar{w}_{1}\left(M^{n}\right) \cup \bar{w}_{n-1}\left(M^{n}\right),\left[M^{n}\right]_{2}\right\rangle\left(\in \mathbf{Z}_{2}\right)$ is a cobordism invariant of $M^{n}$.

Here we give an easy application of Theorem 1.4 to the study of maps with fold singularities (see [7, 17]). For a smooth map $f: M^{n} \rightarrow N^{p}(n \geq p)$, we denote by $S(f)$ the singular set of $f$. Recall that a component $S$ of $S(f)$ is said to be a fold component if every point of $S$ is a fold singularity of $f$. Note that a fold component is always a non-singular $(p-1)$-dimensional closed submanifold of $M^{n}$.

Proposition 5.4. Let $f: S^{4 m} \rightarrow \mathbf{R}^{2 m+1}$ be a smooth map and $S \subset S(f)$ a fold component. Then we have

$$
\left\langle\bar{w}_{1}(S) \cup \bar{w}_{2 m-1}(S),[S]_{2}\right\rangle \equiv 0 .
$$

Furthermore, we have

$$
S \cdot S \equiv 0 \quad(\bmod 4),
$$

where $S \cdot S$ denotes the self-intersection number of $S$ in $S^{4 m}$.

Proof. First consider the case where $m=1$. Then $S$ is a closed surface. When $S$ is orientable, we always have

$$
\left\langle\bar{w}_{1}(S) \cup \bar{w}_{1}(S),[S]_{2}\right\rangle \equiv \chi(S) \equiv 0 \quad(\bmod 2) .
$$

When $S$ is non-orientable, $S$ is an indefinite fold component and hence $S \cdot S=0$ by [17]. Combining this with Theorem 1.4, we have

$$
\left\langle\bar{w}_{1}(S) \cup \bar{w}_{1}(S),[S]_{2}\right\rangle \equiv 0 .
$$

(Here we can regard $S$ as an embedded submanifold in $\mathbf{R}^{4 m}=S^{4 m}-\{*\}$.) Note that, in this case, we always have $S \cdot S=0$.

Next we consider the case where $m \geq 2$. Since $f \mid S: S \rightarrow \mathbf{R}^{2 m+1}$ is an immersion, we see that $\bar{w}_{i}(S)=0\left({ }^{\forall} i \geq 2\right)$. Thus we have

$$
\left\langle\bar{w}_{1}(S) \cup \bar{w}_{2 m-1}(S),[S]_{2}\right\rangle \equiv 0 .
$$

Then by Theorem 1.4, we have

$$
S \cdot S \equiv 0 \quad(\bmod 4) .
$$

This completes the proof. II 


\section{Lifting PROBlem AND PROOF OF THEOREM 1.8}

Let $g: M^{n} \rightarrow \mathbf{R}^{2 n-1}$ be a stable map (or an immersion with normal crossings). Suppose that $\pi: \mathbf{R}^{2 n} \rightarrow \mathbf{R}^{2 n-1}$ is a projection. In this section, we consider the problem of finding a lift $f: M^{n} \rightarrow \mathbf{R}^{2 n}$ of $g$ which is an embedding, where $f$ is a lift of $g$ if $g=\pi \circ f$. Recall that we have already a result in this direction, i.e., Corollary 1.2, which is a direct consequence of a result of Szücs.

First we prove Theorem 1.5.

Proof of Theorem 1.5. We may assume that $\pi$ is the standard projection defined by $\pi\left(x_{1}, \cdots, x_{n-1}, x_{n}\right)=\left(x_{1}, \cdots, x_{n-1}\right)$. First we prove part (1). We construct a smooth function $h: M^{n} \rightarrow \mathbf{R}$ with the following properties:

(a) if $g(x)=g(y)$ for $x \neq y$, then $h(x) \neq h(y)$ or when $h(x)=h(y)$ we have $\operatorname{Im} d(g, h)_{x} \oplus \operatorname{Im} d(g, h)_{y}=T_{h(x)} \mathbf{R}^{2 n}$, and

(b) if rank $d g_{x}<n$, then $\operatorname{dim} d h_{x}\left(\right.$ ker $\left.d g_{x}\right)=1$.

Take a point $p \in S(g)$. Recall that, in a neighborhood of $p, g$ is of the following normal form:

$$
y_{1} \circ g=x_{1}^{2}, y_{i} \circ g=x_{i}(i=2, \cdots, n), y_{n+j} \circ g=x_{1} x_{j+1}(j=1, \cdots, n-1) .
$$

Then we define $h\left(x_{1}, \cdots, x_{n}\right)=x_{1}$ in this neighborhood. Then it is not difficult to see that $h$ satisfies the above conditions (a) and (b) over the neighborhood of $S(g)$. Next we extend this $h$ to a neighborhood of $\overline{A(g)}$ as follows. It is easy to find a smooth function on a neighborhood of $\overline{A(g)}$ such that, for $x, y \in \overline{A(g)}$ with $g(x)=g(y)$ and $x \neq y$, we have $h(x) \neq h(y)$, or $h(x)=h(y)$ and there exist $v \in T_{x}(\overline{A(g)})$ and $w \in T_{y}(\overline{A(g)})$ with $0 \neq d g_{x}(v)=d g_{y}(w) \in T_{g(x)}(g(\overline{A(g)}))$ and $d h_{x}(v) \neq d h_{y}(w)$. Then we extend the above constructed smooth function to a smooth function defined on the whole of $M^{n}$ arbitrarily. Then $f=(g, h): M^{n} \rightarrow$ $\mathbf{R}^{2 n-1} \times \mathbf{R}=\mathbf{R}^{2 n}$ is a desired immersion with normal crossings.

Next we prove part (2). Suppose that there exists an embedding $f: M^{n} \rightarrow \mathbf{R}^{2 n}$ such that $\pi \circ f=g$ and that $g$ has a non-trivial double point circle $C \subset \overline{B(g)}$. Since $C$ is non-trivial, there exists a diffeomorphism $\psi: g^{-1}(C) \rightarrow S^{1}$ such that $g(x)=g(y)$ if and only if $\psi(x)= \pm \psi(y)$ for $x, y \in g^{-1}(C)$, where $S^{1}$ is the unit circle in $\mathbf{R}^{2}$. Then the composite $\varphi=p_{2 n} \circ f \circ \psi^{-1}: S^{1} \rightarrow \mathbf{R}$ is a smooth function such that $\varphi(x) \neq \varphi(-x)$ for every $x \in S^{1}$, since $f$ is an embedding, where $p_{2 n}: \mathbf{R}^{2 n} \rightarrow \mathbf{R}$ is the projection defined by $p_{2 n}\left(x_{1}, \cdots, x_{2 n}\right)=x_{2 n}$. However, it is an easy consequence of the intermediate-value theorem that there does not exist such a continuous function on $S^{1}$. Hence, if an embedding $f$ with $g=\pi \circ f$ exists, $g$ cannot have a non-trivial double point circle.

Conversely, suppose that $g$ has no non-trivial double point circles. In the proof of part (1), it suffices to construct a smooth function $h: M^{n} \rightarrow \mathbf{R}$ satisfying the following condition $\left(\mathrm{a}^{\prime}\right)$ and the previous condition $(\mathrm{b})$ :

$\left(\mathrm{a}^{\prime}\right)$ if $g(x)=g(y)$ for $x \neq y$, then $h(x) \neq h(y)$.

We construct $h$ in a neighborhood of $S(g)$ as follows. For each arc component $J \subset \overline{B(g)}$, on the neighborhoods of the two Whitney umbrella points $g^{-1}(\partial J)$, we define $h\left(x_{1}, \cdots, x_{n}\right)=x_{1}$ or $-x_{1}$ so that we can construct $h$ on $g^{-1}(J)$ with the property $\left(\mathrm{a}^{\prime}\right)$. For a circle component $C \subset \overline{B(g)}$, we can construct $h$ on $g^{-1}(C)$ with the property $\left(\mathrm{a}^{\prime}\right)$ easily, since $g^{-1}(C)$ is disconnected. Then extend this smooth function as in the proof of part (1) and consider $f=(g, h)$. This completes the proof of part (2). 
Now we prove part (3). Recall that, by Proposition 2.5 we have $e(f)=p(f)-$ $n(f)$, where $p(f)$ (resp. $n(f)$ ) is the number of positive (resp. negative) Whitney umbrella points of $g$. Furthermore every arc component $J \subset \overline{B(g)}$ must be trivial by Lemma 4.1, since $f$ has no double points. Hence, the signs of the two Whitney umbrella points $g^{-1}(\partial J)$ coincide if and only if the normal bundle of $g^{-1}(J)$ in $M^{n}$ is non-trivial. We denote by $\beta_{+}$(resp. $\beta_{-}$) the number of arc components $J$ such that the normal bundle of $g^{-1}(J)$ in $M^{n}$ is non-trivial and that the signs of the two Whitney umbrella points $g^{-1}(\partial J)$ are both positive (resp. negative). Then we have

$$
\beta_{+}+\beta_{-}=\beta(g)
$$

and

$$
e(f)=2 \beta_{+}-2 \beta_{-} .
$$

Thus we have $e(f) \in\{-2 \beta(g),-2 \beta(g)+4, \cdots, 2 \beta(g)\}$. We can prove the realization result by using an argument similar to that for part (2). We have only to change $h$ on $g^{-1}(J)$ properly for each arc component $J$. This completes the proof of Theorem 1.5. \|

Remark 6.1. Consider the case $n=2$. Suppose that $f: M^{2} \rightarrow \mathbf{R}^{4}$ is an embedding such that $M^{2}$ is non-orientable and that $g=\pi \circ f$ does not have a triple point. If $J$ is an arc component of $\overline{B(g)}$ such that the normal bundle of $g^{-1}(J)$ in $M^{2}$ is nontrivial, then the tubular neighborhood of $g^{-1}(J)$ is diffeomorphic to the Möbius band. Since $g^{-1}(J) \cap g^{-1}\left(J^{\prime}\right)=\emptyset$ for distinct arc components $J$ and $J^{\prime}$, we see that the number $\beta(g)$ of arc components $J$ such that the normal bundle of $g^{-1}(J)$ is non-trivial cannot exceed the non-orientable genus of $M^{2}$. Then by Theorem 1.5 (3), we have

$$
-4+2 \chi\left(M^{2}\right) \leq e(f) \leq 4-2 \chi\left(M^{2}\right),
$$

which is the well-known Whitney conjecture, where $\chi$ denotes the Euler characteristic. Note that the above inequality has been proved for general embeddings by Massey [14] and also by Kamada [9]. The proofs by Massey and Kamada use the double branched covering of $S^{4}=\mathbf{R}^{4} \cup\{\infty\}$ branched along $f\left(M^{2}\right)$ and they are completely different from our approach. Our method would probably give a new geometric proof of the Whitney conjecture. Note that Theorem 1.5 (1) holds even if $g$ has a triple point, while results corresponding to Theorem 1.5 (2) and (3) do not in general. See Remarks 6.2 and 5.1.

Now we prove Theorem 1.8 in the introduction.

Proof of Theorem 1.8. First we prove part (1). Suppose that $n \geq 4$. Then the stable map $g: M^{n} \rightarrow \mathbf{R}^{2 n-1}$ does not have a triple point. Hence, by Theorem 1.5, we have only to show that $\delta(g)=0$, which follows immediately from Lemma 4.9 (1) and the assumption that $M^{n}$ is orientable.

Next we prove part (2). First we consider the case $n>2$. When $M^{n}$ is nonorientable and $n$ is even, take an orientation reversing simple closed curve $C$ in $M^{n}$. Otherwise take an orientation preserving simple closed curve $C$ in $M^{n}$. Then it is easy to construct an immersion $\xi: N(C) \rightarrow \mathbf{R}^{2 n-1}$ such that the double point set $\xi(A(\xi))$ coincides with $\xi(C)$ and that $\xi \mid C: C \rightarrow \xi(C)$ is a non-trivial double cover, where $N(C)$ is the tubular neighborhood of $C$ in $M^{n}$. (See the proof of Lemma 4.9 (1).) Since $\mathbf{R}^{2 n-1}$ is contractible, $\xi$ can be extended to a smooth 
map $g_{1}: M^{n} \rightarrow \mathbf{R}^{2 n-1}$. By changing $g_{1}$ outside $N(C)$, we obtain a stable map $g_{2}: M^{n} \rightarrow \mathbf{R}^{2 n-1}$. Since $n>2, \xi(C)$ remains to be a non-trivial double point circle of $g_{2}$. Then, by the method of Whitney [24], we can eliminate the Whitney umbrella points of $g_{2}$ without changing the map on $N(C)$. Let this map be denoted by $g: M^{n} \rightarrow \mathbf{R}^{2 n-1}$. Note that, since $n>2, g$ has no triple point. Then by Theorem 1.5 (2), there exists no embedding $f: M^{n} \rightarrow \mathbf{R}^{2 n}$ such that $g=\pi \circ f$, since $g$ has a non-trivial double point circle.

Next consider the case $n=2$. Then $M^{2}$ is a non-orientable surface and is diffeomorphic to the connected sum of some, say $r$, copies of the projective plane $\mathbf{R} P^{2}$. Note that there exists an immersion with normal crossings $g_{1}: \mathbf{R} P^{2} \rightarrow \mathbf{R}^{3}$. Then, since the Euler characteristic of $\mathbf{R} P^{2}$ is odd, there exists no embedding $f_{1}: \mathbf{R} P^{2} \rightarrow \mathbf{R}^{4}$ such that $\pi \circ f_{1}=g_{1}$, by Theorem 1.4. (See also Theorem 1.7 and [6, Remark (p.610)].) Now let $g: M^{2} \rightarrow \mathbf{R}^{3}$ be the immersion with normal crossings which is constructed by the connected sum of $r$ copies of $g_{1}$. Then it is easy to see that $g$ cannot be lifted to an embedding into $\mathbf{R}^{4}$, since, if it is lifted, $g_{1}$ is also lifted, and this leads to a contradiction. This completes the proof of Theorem 1.8. $\|$

Remark 6.2. Note that Theorem 1.8 (1) does not hold for $n=2$. In fact, Giller [6, p.600] has given an immersion with normal crossings $g: S^{2} \rightarrow \mathbf{R}^{3}$ which cannot be lifted to an embedding into $\mathbf{R}^{4}$. Furthermore, Giller has given a characterization of immersions with normal crossings of orientable surfaces into $\mathbf{R}^{3}$ which can be lifted to embeddings into $\mathbf{R}^{4}[\mathbf{6}$, Proposition 4].

\section{Canceling Whitney umbrellas and proof of Theorem 1.7}

Before proving Theorem 1.7, we prove the following lemma, which is a direct generalization of a result of Carter and Saito [5] for $n=2$.

Lemma 7.1. Suppose that $f: M^{n} \rightarrow \mathbf{R}^{2 n}$ is an embedding such that $g=\pi \circ f$ is stable, where $\pi: \mathbf{R}^{2 n} \rightarrow \mathbf{R}^{2 n-1}$ is a projection, $n$ is even, $\mathbf{R}^{2 n}$ is oriented and $M^{n}$ is connected. Then a pair of oppositely signed Whitney umbrella points of $g$ are canceled by an isotopy of $f$ without creating any new Whitney umbrella point.

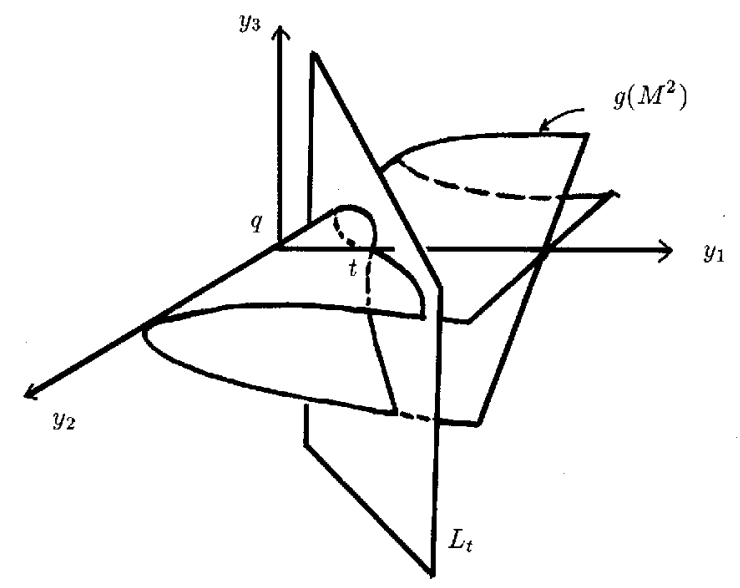

FiguRE 5. $n=2$ 


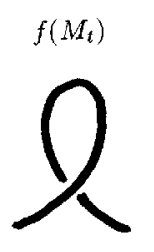

$t>0$
$f\left(M_{0}\right)$

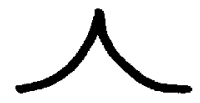

$t=0$
$f\left(M_{t}\right)$

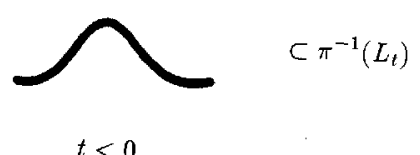

FiguRE 6

Proof. Let $p \in M^{n}$ be a Whitney umbrella point of $g$ and set $q=g(p)$. We choose local coordinates $\left(x_{1}, \cdots, x_{n}\right)$ centered at $p$ and $\left(y_{1}, \cdots, y_{2 n-1}\right)$ centered at $q$ for which $g$ has the normal form as in $\S 2$. For $t \in \mathbf{R}$ and $\left(a_{2}, \cdots, a_{n}\right) \in \mathbf{R}^{n-1}-\{0\}$, we set

$$
L_{t}=\left\{\left(y_{1}, \cdots, y_{2 n-1}\right): y_{1}+a_{2} y_{2}+\cdots+a_{n} y_{n}=t\right\}
$$

and $M_{t}=g^{-1}\left(g\left(M^{n}\right) \cap L_{t}\right) \subset M^{n}$ (see Figure 5). Then we see easily that $M_{t}=$ $\left\{\left(x_{1}, \cdots, x_{n}\right): x_{1}^{2}+a_{2} x_{2}+\cdots+a_{n} x_{n}=t\right\}$. Since $\left(a_{2}, \cdots, a_{n}\right) \neq 0$, we may assume $a_{n} \neq 0$, for example. Then the map defined by $\left(x_{1}, \cdots, x_{n}\right) \mapsto\left(x_{1}, \cdots, x_{n-1}, x_{1}^{2}+\right.$ $\left.a_{2} x_{2}+\cdots+a_{n} x_{n}-t\right)$ is a diffeomorphism. Set $\left(X_{1}, \cdots, X_{n}\right)=\left(x_{1}, \cdots, x_{n-1}, x_{1}^{2}+\right.$ $\left.a_{2} x_{2}+\cdots+a_{n} x_{n}-t\right)$, which is another local coordinate centered at $p$. Then we have $M_{t}=\left\{X_{n}=0\right\}$. Elementary but tedious calculations show the following:

(a) the map $g \mid M_{t}$ is an immersion if $t \neq 0$, and when $t=0$, it is an immersion except at $\left(X_{1}, \cdots, X_{n-1}\right)=0$, and

(b) when $t>0$, for $\left(X_{1}, \cdots, X_{n-1}\right),\left(X_{1}^{\prime}, \cdots, X_{n-1}^{\prime}\right) \in M_{t}, g\left(X_{1}, \cdots, X_{n-1}\right)$ $=g\left(X_{1}^{\prime}, \cdots, X_{n-1}^{\prime}\right)$ if and only if $X_{1}, X_{1}^{\prime}= \pm \sqrt{t}, X_{2}=\cdots=X_{n-1}=X_{1}^{\prime}=\cdots=$ $X_{n-1}^{\prime}=0$ and $g \mid M_{t}$ is an immersion with normal crossings, and when $t<0, g \mid M_{t}$ is an embedding.

In other words, we can consider $g$, in a neighborhood of $p$, as a projection move of the isotopy $f \mid M_{t}(t \in(-\varepsilon, \varepsilon)$ ), where $\varepsilon>0$ is sufficiently small (see Figure 6 ).

Now we suppose $n>2$. (Recall that the case $n=2$ has been proved by Carter and Saito [5].) The choice of $L_{t}$ corresponds to the choice of $\left(a_{2}, \cdots, a_{n}\right) \in \mathbf{R}^{n-1}-$ $\{0\}$, where $\mathbf{R}^{n-1}-\{0\}$ is connected. Hence the choice of $L_{t}$ is essentially unique. (Note that, when $n=2$, we have essentially two choices of $L_{t}$.)

Now take two Whitney umbrella points $p, p^{\prime} \in M^{n}$ with opposite signs. Since $M^{n}$ is connected we can connect $p$ and $p^{\prime}$ by a simple closed curve $\alpha$. Since $n>2$, we may assume that $\alpha \cap \overline{A(g)}=\left\{p, p^{\prime}\right\}$. By sliding the Whitney umbrella points along this curve, we may assume that $p$ and $p^{\prime}$ are sufficiently close to each other, changing $f$ by an isotopy. If we consider the map $g$ in neighborhoods of $p$ and $p^{\prime}$ as projection moves, we see that they correspond to moves opposite to each other. This is because the choice of $L_{t}$ at a Whitney umbrella point is essentially unique and the signs of the Whitney umbrella points $p$ and $p^{\prime}$ are opposite. Then we can change $f$ in a neighborhood of $\alpha$ by an isotopy so that the two Whitney umbrella points are eliminated. For details see Figures 7 and 8 . This completes the proof of Lemma 7.1. $\|$

Since pairs of oppositely signed Whitney umbrella points can be eliminated successively by Lemma 7.1, we have proved Theorem 1.6 in the introduction, which is a direct generalization of the main theorem of $[\mathbf{5}]$. 

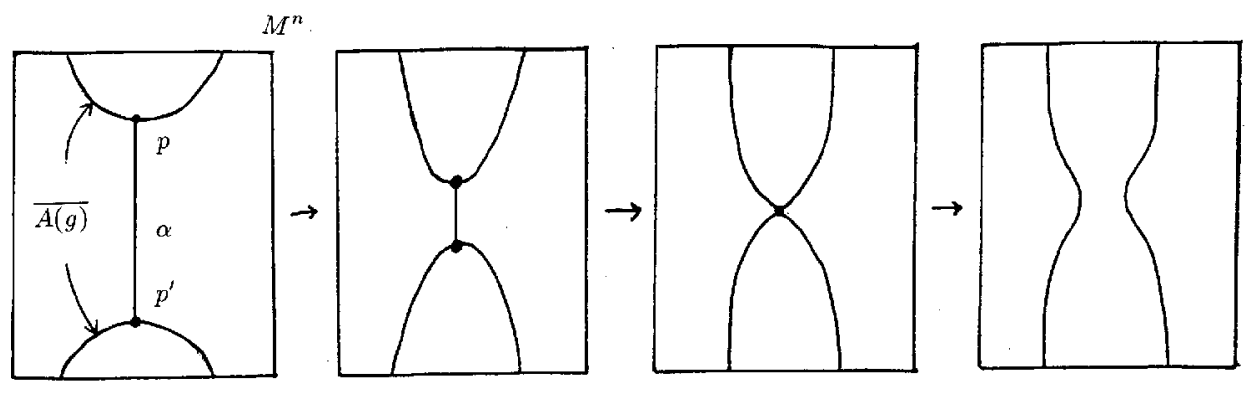

FiguRE 7

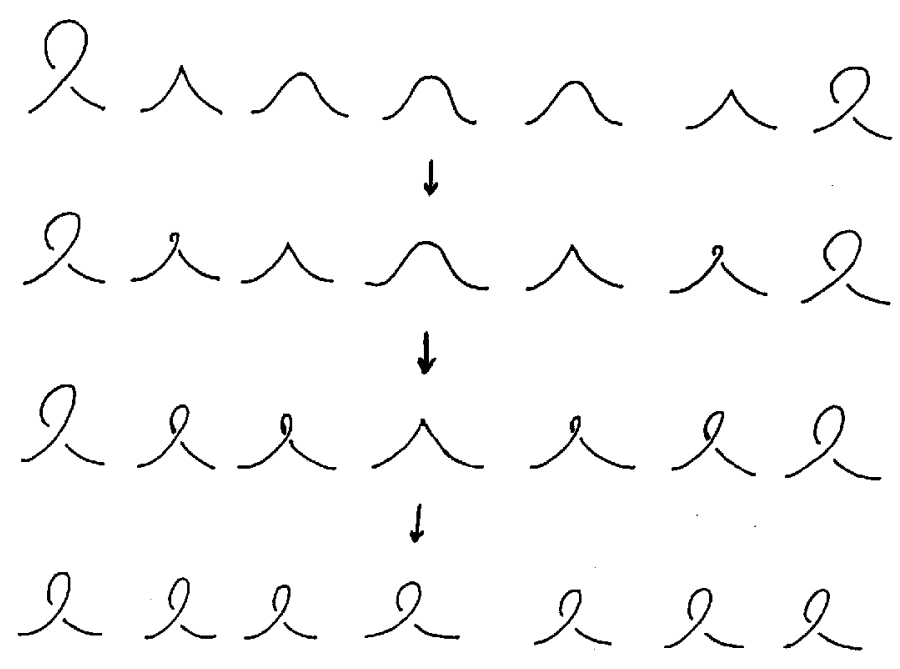

Figure 8

Remark 7.2. Hirsch [8, Theorem 6.4] showed that if $M^{n}$ is immersed into $\mathbf{R}^{m+r}$ $(m>n)$ with a transversal $r$-field, then it can be immersed into $\mathbf{R}^{m}$. (Moreover, such an immersion into $\mathbf{R}^{m+r}$ is regularly homotopic to one in $\mathbf{R}^{m}$.) Theorem 1.6 gives another proof of this result for $n$ even, $r=1$, and $m=2 n-1$ as follows. Suppose that $f: M^{n} \rightarrow \mathbf{R}^{2 n}$ is an immersion with a transversal 1-field. Then $f$ is regularly homotopic to an immersion with normal crossings $f_{1}: M^{n} \rightarrow \mathbf{R}^{2 n}$ with a transversal 1-field. Then we see that $e\left(f_{1}\right)=0$, and by Theorem 1.6 , we can change $f_{1}$ by a regular homotopy so that it has no Whitney umbrella points when composed with the projection $\pi: \mathbf{R}^{2 n} \rightarrow \mathbf{R}^{2 n-1}$. (Note that, in Lemma 7.1 and Theorem 1.6, $f$ can be an immersion with normal crossings, if we replace the word "isotopy" by "regular homotopy".) Then $g=\pi \circ f: M^{n} \rightarrow \mathbf{R}^{2 n-1}$ is an immersion.

Now we proceed to the proof of Theorem 1.7.

Proof of Theorem 1.7. It is easy to see that parts (1) and (2) are equivalent to each other. Now we prove that part (3) implies part (1). Suppose that there exists an embedding $f: M^{n} \rightarrow \mathbf{R}^{2 n}$ such that $g=\pi \circ f$. Then it is easily seen that the normal bundle of $f$ has a section which is nowhere zero. Hence we have $e(f)=0$ if $n$ is even. 
Furthermore, since $f$ is an embedding, $D(f)=0$. This contradicts the assumption (3) by Theorem 1.4. When $n$ is odd, it is known that $\bar{w}_{1}\left(M^{n}\right) \cup \bar{w}_{n-1}\left(M^{n}\right)=0$ by Massey [13]. Thus, when $n$ is odd, the assumption (3) is not satisfied. Thus we have proved that part (3) implies part (1). Note that this argument is essentially the same as that used in [6, Remark (p. 610)].

Now we prove that part (1) implies part (3). Suppose that

$$
\left\langle\bar{w}_{1}\left(M^{n}\right) \cup \bar{w}_{n-1}\left(M^{n}\right),\left[M^{n}\right]_{2}\right\rangle \equiv 0 .
$$

Choose an arbitrary immersion with normal crossings $f_{1}: M^{n} \rightarrow \mathbf{R}^{2 n}$. We will modify $f_{1}$ so that it becomes an embedding which projects to an immersion into $\mathbf{R}^{2 n-1}$.

First we consider the case where $n$ is even. We will modify $f_{1}$ so that $e\left(f_{1}\right)=0$.

Case 1. When $M^{n}$ is orientable.

Orient $M^{n}$ arbitrarily. Let $I\left(f_{1}\right) \in \mathbf{Z}$ be the self-intersection number of $f_{1}$ defined by Whitney $[\mathbf{2 3}]$. Then we have the following.

Lemma 7.3. $e\left(f_{1}\right)+2 I\left(f_{1}\right)=0$.

Proof. Consider the intersection number of $f_{1}\left(M^{n}\right)$ and its push-off. Then every self-intersection point of $f_{1}$ contributes by twice the sign of the self-intersection. Thus we have

$$
\left(f_{1}\right)_{*}\left[M^{n}\right] \cdot\left(f_{1}\right)_{*}\left[M^{n}\right]=e\left(f_{1}\right)+2 I\left(f_{1}\right),
$$

where $\left[M^{n}\right] \in H_{n}\left(M^{n} ; \mathbf{Z}\right)$ is the fundamental class of $M^{n}$, and the left hand side denotes the intersection number in $\mathbf{R}^{2 n}$. Since $H_{n}\left(\mathbf{R}^{2 n} ; \mathbf{Z}\right)=0$, we have the conclusion. This completes the proof. \|

By the method of Whitney [23], we can modify $f_{1}$ so that $I\left(f_{1}\right)=0$. For this immersion $f_{1}$, we have $e\left(f_{1}\right)=0$ by Lemma 7.3.

Case 2. When $M^{n}$ is non-orientable.

First, by Theorem 1.4 and our assumption, we have

$$
\frac{e\left(f_{1}\right)}{2}+D\left(f_{1}\right) \equiv 0 \quad(\bmod 2) .
$$

By the method of Whitney, we can modify $f_{1}$ so that $D\left(f_{1}\right)$ is increased by one and that $e\left(f_{1}\right)$ is changed by \pm 2 (see the proof of Lemma 7.3). Thus we may assume that $e\left(f_{1}\right)=0$ and $D\left(f_{1}\right)$ is even.

Now assume that $n \geq 3$. Then by Whitney's trick [23], we can change $f_{1}$ by a regular homotopy to an embedding $f_{2}: M^{n} \rightarrow \mathbf{R}^{2 n}$. Note that $e\left(f_{1}\right)=e\left(f_{2}\right)=0$. Then by Theorem 1.6, we can change $f_{2}$ by an isotopy to an embedding $f: M^{n} \rightarrow$ $\mathbf{R}^{2 n}$ such that $g=\pi \circ f$ is an immersion. This contradicts our assumption (1).

When $n=2$, since $\left\langle\bar{w}_{1}\left(M^{2}\right) \cup \bar{w}_{1}\left(M^{2}\right),\left[M^{2}\right]_{2}\right\rangle \equiv 0$, we see that the Euler characteristic $\chi\left(M^{2}\right)$ of $M^{2}$ is even. When $M^{2}$ is orientable, $M^{2}$ can be embedded into $\mathbf{R}^{3}$, which can easily be lifted to an embedding into $\mathbf{R}^{4}$. This is a contradiction. When $M^{2}$ is non-orientable, $M^{2}$ is diffeomorphic to the connected sum of some copies of the Klein bottle. Then consider the immersion $g: M^{2} \rightarrow \mathbf{R}^{3}$ with normal crossings without triple points as in Figure 9. Then, since every double point circle 


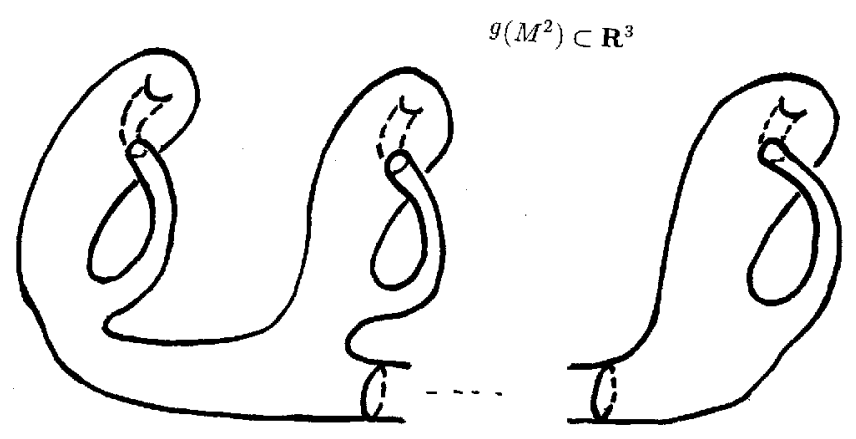

FIGURE 9

of $g$ is trivial, it can be lifted to an embedding into $\mathbf{R}^{4}$ by Theorem 1.5 (2). This is also a contradiction.

Now we consider the case where $n$ is odd $(n \geq 3)$. By [23], there exists an embedding $f_{1}: M^{n} \rightarrow \mathbf{R}^{2 n}$. Let $e(\in \mathbf{Z})$ be the intersection number of $f_{1}\left(M^{n}\right)$ and its push-off into the positive direction of ker $\pi$. Then by an argument as in the proof of Proposition 2.5, we see that

$$
e=-\left(p\left(f_{1}\right)-n\left(f_{1}\right)\right)
$$

where $p\left(f_{1}\right)$ and $n\left(f_{1}\right)$ are the numbers of positive and negative Whitney umbrella points respectively. Here we orient $\mathbf{R}^{2 n-1}$ properly and then the sign of a Whitney umbrella point is defined in exactly the same way as in the case where $n$ is even (see §2). On the other hand, it is known that $e$ is always equal to zero, when $n$ is odd. Then the rest of the argument is exactly the same as that for $n$ even. This completes the proof of Theorem 1.7. \|

\section{REFERENCES}

1. M. Audin, Fibrés normaux d'immersions en dimension double, points doubles d'immersions lagrangiennes et plongements totalement réels, Comment. Math. Helv. 63 (1988), 593-623. MR 89m:57032

2. M. Audin, Quelques remarques sur les surfaces lagrangiennes, J. Geom. Phys. 7 (1990), 583598. MR 92i:57022

3. T. F. Banchoff, Double tangency theorems for pairs of submanifolds, Geometry Symposium Utrecht 1980 (Looijenga, Siersma and Takens, eds.), Lect. Notes in Math., vol. 894, SpringerVerlag, Berlin and New York, 1981, pp. 26-48. MR 83h:53005

4. S. J. Blank and C. Curley, Desingularizing maps of corank one, Proc. Amer. Math. Soc. 80 (1980), 483-486. MR 82e:57017

5. J. S. Carter and M. Saito, Canceling branch points on projections of surfaces in 4-space, Proc. Amer. Math. Soc. 116 (1992), 229-237. MR 93i:57029

6. C. A. Giller, Towards a classical knot theory for surfaces in $\mathbf{R}^{4}$, Illinois J. Math. 26 (1982), 591-631. MR 84c:57011

7. M. Golubitsky and V. Guillemin, Stable mappings and their singularities, Grad. Texts in Math. no.14, Springer-Verlag, New York-Heidelberg-Berlin, 1973. MR 49:6269

8. M. W. Hirsch, Immersions of manifolds, Trans. Amer. Math. Soc. 93 (1959), 242-276. MR 22:9980

9. S. Kamada, Non-orientable surfaces in 4-space, Osaka J. Math. 26 (1989), 367-385. MR 91g:57022 
10. J. Lannes, Sur les immersions de Boy, Algebraic Topology, Aarhus 1982, edited by Madsen and Oliver, Lecture Notes in Math. 1051, Springer-Verlag, Berlin-Heidelberg-New York-Tokyo, 1984, pp. 263-270. MR 86d:57015

11. Li Bang-He, Generalization of the Whitney-Mahowald Theorem, Trans. Amer. Math. Soc. 346 (1994), 511-521.

12. M. Mahowald, On the normal bundle of a manifold, Pacific J. Math. 14 (1964), 1335- 1341. MR 31:757

13. W. S. Massey, On the Stiefel-Whitney classes of a manifold, Amer. J. Math. 82 (1960), 92-102. MR 22:1918

14. W. S. Massey, Proof of a conjecture of Whitney, Pacific J. Math. 31 (1969), 143-156. MR 40:3570

15. J. Mather, Generic projections, Ann. of Math. 98 (1973), 226-245. MR 50:14835

16. F. Ronga, 'La class duale aux points double' d'une application, Compositio Math. 27 (1973), 223-232. MR 52:11936

17. O. Saeki, Notes on the topology of folds, J. Math. Soc. Japan 44 (1992), 551-566. MR 93f: 57037

18. A. Szücs, Surfaces in $\mathbf{R}^{3}$, Bull. London Math. Soc. 18 (1986), 60-66. MR 88a:57068

19. A. Szücs, Note on double points of immersions, Manuscripta Math. 76 (1992), 251-256. MR 93h:57047

20. R. Thom, Quelques propriétés globales des variétés différentiables, Comment. Math. Helv. 28 (1954), 17-86. MR 15:890a

21. H. Whitney, On the topology of differentiable manifolds, Lectures in Topology, Michigan Univ. Press, 1940. MR 3:133a

22. H. Whitney, The general type of singularity of a set of $2 n-1$ smooth functions of $n$ variables, Duke Math. J. 10 (1943), 161-172. MR 4:193b

23. H. Whitney, The self-intersection of a smooth n-manifold in $2 n$-space, Ann. of Math. 45 (1944), 220-246. MR 5:273g

24. H. Whitney, The singularities of a smooth $n$-manifold in $(2 n-1)$-space, Ann. of Math. 45 (1944), 247-293. MR 5:274a

25. Y. Yamada, An extension of Whitney's congruence, Osaka J. Math. 32 (1995), 185-192.

Department of Mathematics, Faculty of Science, Hiroshima University, HigashiHiROSHIMA 739, JAPAN

E-mail address: saeki@top2.math.sci.hiroshima-u.ac.jp

Department of General Education, Kochi National College of Technology, NanKOKU CITY, KOCHI 783, JAPAN

E-mail address: sakuma@cc.kochi-ct.ac.jp 